Onkologe 2010 $\cdot 16: 643$

DOI 10.1007/s00761-010-1873-z

(c) Springer-Verlag 2010
G. Brabant ${ }^{1} \cdot$ M. Bamberg ${ }^{2}$

${ }^{1}$ Dept. of Endocrinology, Christie Hospital, Manchester

2Universitätsklinik für Radioonkologie, Tübingen

\title{
Update zur Behandlung des Schilddrüsenkarzinoms
}

In den letzten Jahren hat die Behandlung von Schilddrüsenkarzinomen insbesondere aufgrund der internationalen, in Leitlinien niedergelegten Vorgehensweise eine bessere Standardisierung erfahren. Neben dem weiter differenzierten chirurgischen Vorgehen und wichtigen Änderungen in der nuklearmedizinischen Nachsorge sind vor allem durch eine Reihe internationaler Studien zum Einsatz von Rezeptortyrosinkinaseninhibitoren neue Optionen zur Chemotherapie bei aggressiven Karzinomen in den Vordergrund gerückt, welche aber weiterhin durch die externe Strahlentherapie wirksam unterstützt werden.

Die aktuelle Übersicht von K.W. Schmid evaluiert die deutlichen Fortschritte in der molekularen Klassifikation von Schilddrüsenkarzinomen und diskutiert die praktischen Probleme, welche aus den Veränderungen der Klassifikation durch die WHO im Alltag entstehen.

Die Differenzierung von Schilddrüsenknoten in benigne und maligne stellt eines der großen ungelösten Probleme dar. Basierend auf den der kürzlich erschienenen europäischen Leitlinien geben W. Karges und G. Brabant eine pragmatische Anleitung zur Stratifizierung und diskutieren die diagnostischen Ansätze zur Sicherung der Diagnose unter direktem Bezug auf die deutsche Situation.

Das chirurgische Vorgehen konnte nicht zuletzt durch die systematischen Untersuchungen aus der Hallenser Arbeitsgruppe weiter differenziert werden. In ihrem Übersichtsbeitrag beschreiben H. Dralle, K. Lorenz, A. Machens, M. Brauckhoff und P. Nguyen-Thanh eine klares Konzept zum risikostratifizierten chirurgischen Vorgehen. Insbesondere die neuen Ansätze bei der chirurgischen Behandlung von Hochrisikopatienten lassen eine signifikante Verbesserung des Langzeitüberlebens erwarten.

M. Dietlein, M. Luster und C. Reiners diskutieren den Status quo der nuklearmedizinischen Nachsorge von Schilddrüsenkarzinomen. Insbesondere die Einführung von rekombinantem humanem TSH hat zu einer wesentlichen Verbesserung der Lebensqualität von Patienten in der Nachsorge geführt. Die Implikationen dieser neuen Diagnostikmodalität gegenüber dem herkömmlichen Ansatz, Schilddrüsenhormone passager zu entziehen, werden auch unter finanziellen Gesichtspunkten kritisch diskutiert.

Die Erfolgsraten der herkömmlichen chemotherapeutischen Ansätze beim Schilddrüsenkarzinom sind bislang wenig überzeugend. Kürzlich wurden mehrere neue Substanzen aus der Klasse der Tyrosinkinaseinhibitoren beschrieben, welche sehr vielversprechende Resultate bei den aggressiven, gegenwärtig kaum beeinflussbaren Formen des Schilddrüsenkarzinoms zeigen. Der Review von A. Matuszczyk und K. Mann evaluiert diese neuen Ergebnisse und gibt Referenzen zu den gegenwärtig laufenden Studien.

Der Wert der externen Strahlentherapie gerade bei den aggressiven Schilddrüsenkarzinomen wird im Beitrag von $D$. Vordermark, T. Pelz und F. Sieker kritisch gewürdigt. Der Wert der Primärtumorbestrahlung ist durch neue Untersuchungen besser einzuschätzen, während die ex- terne Radiatio bei Fernmetastasen weiter breitere Anwendung findet.

Allen Autoren gilt besonderer Dank für ihre aktuellen Übersichten, welche in hohem Maße pragmatische Gesichtspunkte zu berücksichtigen suchen.

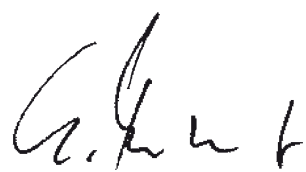

Für die Herausgeber des Schwerpunktheftes
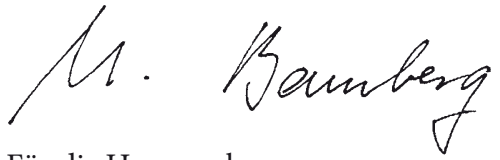

Für die Herausgeber

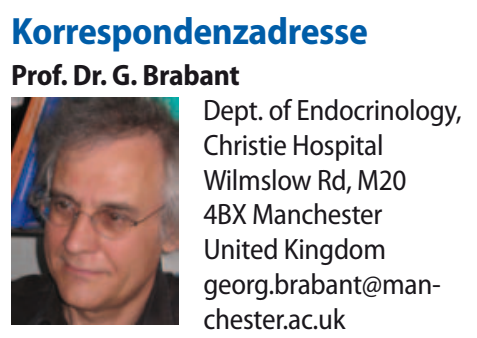

